\title{
THE PATHOLOGICAL EFFECTS PRODUCED BY SERA OF ANIMALS IMMUNIZED WITH FOREIGN NERVOUS OR SPLENIC TISSUE
}

\author{
PART I : INTRACISTERNAL INJECTION OF SERUM
}

BY

\section{E. WESTON HURST}

From Imperial Chemical (Pharmaceuticals) Limited, Hexagon House, Blackley, Manchester

There exists one apparent discrepancy between the reports of investigators who, in the opening years of the present century, attempted to produce " neurolytic" or " neurotoxic" sera by immunizing animals with foreign nervous tissue, and those of more recent workers who by rather similar procedures have studied the genesis of the so-called allergic encephalomyelitis.

At a time when the development of various cytolytic sera was a topic of current interest, a few observers (Centanni, 1900 ; Délezenne, 1900 ; Pirone, 1904 ; Armand-Delille, 1906 ; Schmidt, 1906) injected animals with large doses of foreign nervous tissue, thereby obtaining sera which when introduced intracerebrally into the species providing the nervous tissue caused convulsions, paralyses, and more or less rapid death. On the other hand, a number of experimenters with " allergic encephalitis", evoked by injections of homologous or heterologous brain-tissue, agree that the sera of affected animals do not reproduce the condition, and apparently do not cause disturbance of any kind when given intraperitoneally, intravenously, or intracisternally (Morgan, 1947 ; Freund, Stern, and Pisani, 1947 ; Kabat, Wolf, and Bezer, 1948 ; Hill, 1949). Moreover, although it has long been known that brain-specific antisera, reacting in the complement-fixation test, can be obtained by immunizing animals with suspensions or alcoholic extracts of foreign brain, the sera in cases of " allergic encephalitis" do not constantly show complement-fixing or other antibodies (see Hurst, 1952).

As mentioned in passing elsewhere (Hurst, 1944), at intervals over the years we have prepared neurotoxic sera in a variety of species; the pathological changes such sera produce under various experimental conditions do not appear ever to have been described in detail.

\section{Experimental}

Immunization of Animals.-In an experiment carried out in 1930, a goat received intramuscularly twice-weekly injections of a very thick suspension of simian brain freed as far as possible from blood and connective tissue. $\vec{\infty}$ There was nothing premeditated in the choice of brain $\omega$ and of animal to be immunized; we merely used wha happened to be available. The intramuscular injection $\rightarrow$ were supplemented at irregular intervals by intravenoug injections of a centrifuged $10 \%$ suspension of braip. Immunization continued for seven months, during whic time the goat lost weight but manifested no symptoms of what is now known as "allergic encephalitis". the end of the period the animal was bled.

Other sera prepared along the same lines betweef $\vec{f}$ 1930 and 1940 were in rabbits and sheep against porcinê of brain, and in rabbits against sheep and monkey brain.

Since it appeared desirable to compare the effects produced by the foregoing sera with those of sera resulting from more modern methods of immunization, we have recently twice repeated the initial experiment in two pairs of goats immunized by the technique of Freund and McDermott (1942).

Over a period of two months the animals received intramuscularly seven doses, each of $10 \mathrm{ml}$., of a waterin-oil emulsion of simian brain with added tubercle bacilli (brain $46 \mathrm{~g}$., phenol-saline $80 \mathrm{ml}$., paraffin oil $50 \mathrm{ml}$., dried tubercle bacilli $100 \mathrm{mg}$., " arlacel A" 20 $\mathrm{ml}$. ). In the early stages the goats lost weight, which they regained later in the period of immunization. They $:$ were bled 14 days after the last injection, and a month later the lymph nodes draining the parts receiving inoculum were collected.

For purposes of comparison, two other goats were similarly immunized with simian spleen and serum collected at the end of two months.

The control sera used in this work were furnished by the same animals before immunization.

None of the sera was markedly haemolytic in a o standard system composed of heat-inactivated serum, $N$ guinea-pig complement, and monkey erythrocytes. Thus 은 the recent anti-brain and anti-spleen sera produced 
complete haemolysis at 1 in 4 , partial hacmolysis at 1 in 8 and 1 in 16, and the merest trace at 1 in 32 ; before being lysed the red cells were agglutinated by the sera, and agglutination without haemolysis continued up to a dilution of 1 in 128 . In the absence of complement the sera agglutinated simian red cells. If, however, the dilutions of the sera were made with inactivated normal goat serum, and the simian erythrocytes suspended in normal monkey serum instead of in saline, haemolysis was produced up to a dilution of 1 in 128 . Normal goat serum was not in itself haemolytic under any of these conditions.

\section{Results}

Intracisternal Injection of Neurotoxic Sera.-The following observations are based on the findings in 12 monkeys ( $M$. mulatta) given the first preparation of goat serum, six monkeys given the recent samples, eight pigs given immune rabbit or sheep serum, and a number of monkeys and sheep receiving various immune sera. The serum injected was always one prepared against the nervous tissue of the recipient species; we have performed no experiments to determine whether a serum neurotoxic for one species possesses similar properties for others. No differences were observed in the effects of the samples of goat serum prepared by the two different techniques at an interval of nearly 20 years.

Under ether or nembutal anaesthesia we removed as much cerebrospinal fluid as possible ( 3 to $5 \mathrm{ml}$.) and replaced it with a slightly smaller volume of warmed immune serum; from the results described below, it is evident that under these conditions much of the inoculum found its way into the most remote parts of the ventricular system. Repetition of the procedure became progressively more difficult, and several monkeys died of trauma inflicted upon the medulla in one of the later attempts. The largest number of doses given was five in 18 days. No localizing signs followed the injections, but the animals obviously suffered from meningeal irritation, and their general condition was visibly worse than that of those receiving the corresponding control serum. This was especially the case with goat serum, which in all experiments seemed to be much more neurotoxic than any other of the sera prepared ; it is probably coincidental that the goat is highly resistant to the development of " allergic encephalitis " (Innes, 1951). The serum prepared in rabbits against pig brain 'was fairly active, while many of the others produced scarcely more severe changcs than did the control sera.

Several monkeys apparently fully recovered from the effects of two or three doses of serum, and allowed to remain without further interference, later developed progressive weakness and died with convulsions. At necropsy, as also in several others given multiple doses, the whole ventricular system was greatly dilated, and we were tempted to suppose that the animals had succumbed to the effects of an acute hydrocephalus. Subsequent discovery, in the brains of some of these, of continuing acute inflammatory changes as long as a week after the last dose of serum suggested the possibility of a progressive condition which was not exclusively even though largely mechanical.

Intracisternal Injection of Anti-spleen Serum.The immediate effects were more severe than those of the anti-brain sera. Doses of $1.5 \mathrm{ml}$. and more of the undiluted serum killed recipients within four hours ; at necropsy the meninges were greatly congested and often exhibited punctate haemorrhages. By diluting the serum with saline we were able to study the remote effects of single or repeated doses.

\section{Patholggical Examination}

Microscopical appearances in the brains of animals receiving neurotoxic goat sera were somewhat diverse, and we shall not describe at length a host of pathological minutiae without direct relevance to the main theme of this paper. The diversity was in part related to the number of doses of serum given and the time of necropsy in relation to the last dose, and in part to the fact that no single dose of serum evoked lesions spread uniformly over the whole area potentially exposed to its action. Thus, some areas of the ventricular system of animals receiving multiple doses showed a summation of the effects of several, others the effects of a recent or more remote single dose, while still other areas were apparently unaffected.

In monkeys receiving a single dose of any of the samples of neurotoxic goat serum, and sacrificed within 24 or 48 hours, lesions were found in two situations, in the meninges and in the ventricular system.

In the former they were usually severe over the cerebellum and brain-stem, the base and lateral surface of the cerebrum, and sometimes on the upper medial surface of the hemisphere. They took the form of a severe and predominantly polymorphonuclear meningitis, with early and light microglial reaction and some polymorphonuclear invasion in the immediately subjacent subpial zone of the most severely affected areas, e.g., the depths of the Sylvian fissure. In other places coagulated fluid (serum) was present and small haemorrhages sometimes occurred. The adventitia of the walls of some main arteries was sparsely infiltrated by leucocytes, but the muscular coat was intact. In some of the veins heavy infiltration continued through the whole thickness of the wall. 


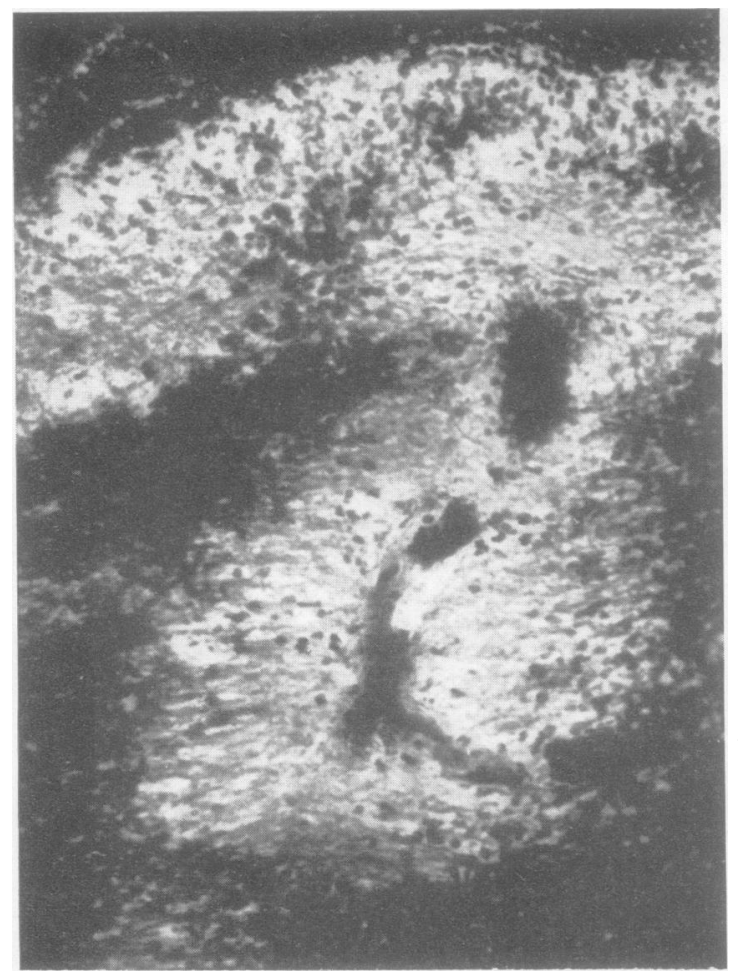

FIG. 1.-Perivascular necrosis around a vessel near an affected part of the lateral ventricle. Single dose of neurotoxic goat serum. $\times 234$.

In the ventricular system lesions were seen at all levels up to the lateral ventricles, but nowhere were they continuous over extensive areas. Over large patches the ependymal cells had disappeared, and in occasional foci immediately beneath, or around a nearby vessel (Fig. 1), the cells were degenerating, their nuclei swollen or pyknotic, and the staining of the background altered as in an early infarct or zone of perivascular necrosis. "Ball" or perivascular haemorrhages were sometimes present. In other areas the tissues beneath the denuded surface were infiltrated with polymorphonuclears or showed early mobilization of the microglia (Fig. 2). The choroid plexuses also were involved in a patchy fashion ; in places the surface epithelium was lost, in others a small tuft might be necrotic (Fig. 3), while the stroma was widely infiltrated with polymorphonuclears. We may perhaps add that cultures for bacteria were always negative.

Repeated doses of serum accentuated the meningitis (Fig. 4). The earlier waves of polymorphonuclears underwent karyorrhexis and were engulfed by phagocytes; mononuclears including a few plasma cells and some granular corpuscles appeared in animals killed some days after a dose, and swelling and proliferation of the cells of the vascular adventitia and of the mesothelial cells of the meninges added to the picture in many brains. Glial enlargement accompanied microglial reaction in a few areas of the subpial zone. Very rarely a small vessel suffered thrombosis. The ventricular system became considerably dilated, and over much of its extent, but never throughout, was lined by a zone of inflammatory tissue (Fig. 5). The changes were not graded in severity according to distance from the cisterna magna, and on the whole were fully as severe in the walls of the lateral as in the fourth ventricle. Just after a dose of serum the cellular exudate in these areas was predominantly polymorphonuclear, and many cells passed into the lumen of the ventricle. Later, microglial phagocytes containing fine sudanophil granules intermingled with mononuclears and perhaps enlarged glial cells, but in some monkeys massed polymorphs occurred in places as late as seven days after the last dose of serum. Adjacent vessels were infiltrated with polymorphonuclears or mononuclears and their adventitial cells were often swollen and proliferating. Other areas from which the ependyma was absent

Fig. 2.-Desquamation of the ependyma of the lateral ventricle. Oedema, light polymorphonuclear infiltration, and early microglial response in the underlying nervous tissue. Single dose of neurotoxic goat serum. $\times 750$.

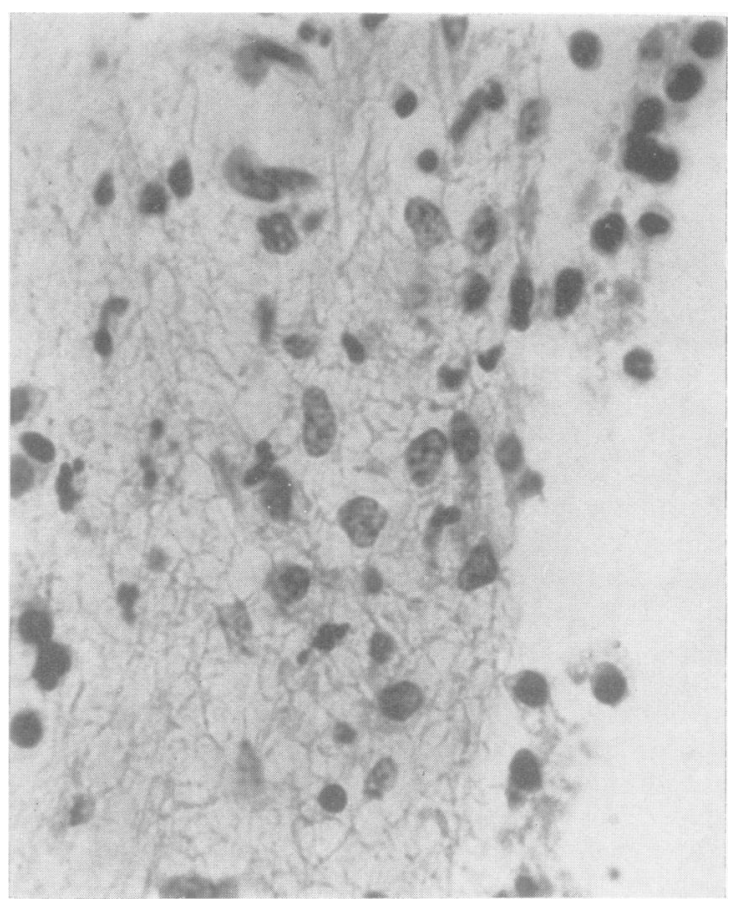


showed principally enlarged glial cells, sometimes in the form of a palisade of multinucleated elements (Fig. 6) ; this appearance commonly obtained in masses of grey matter, e.g., the caudate nuclei, abutting on the ventricles. In one animal patches of the ependyma (possibly regenerated) were several cells deep, and ependyma-like cells were proliferating in rosettes slightly below the surface. In every animal parts of the ventricular surface were apparently normal. Patchy changes in the choroid plexuses have already been outlined; in addition, after several doses of serum, some animals showed in the plexuses large multinucleated giant cells, with or without numerous eosinophils (Fig. 7), and rarely smaller giant cells occurred in parts of the meninges.

Generally speaking, the depth of the cellular zones lining the ventricles was greater in areas of grey matter than where myelinated fibre tracts ran parallel to the surface ; this, no doubt, resulted from mechanical factors. The deepest penetration and most severe lesions often occurred at an angle of the ventricles. While in the more severely affected areas the myelin was destroyed together with other constituents of the tissues, no evidence of selective demyelination existed.

Because the appearance of "allergic encephalitis" has been linked with the development of sensitivity of the tuberculin type (Waksman and

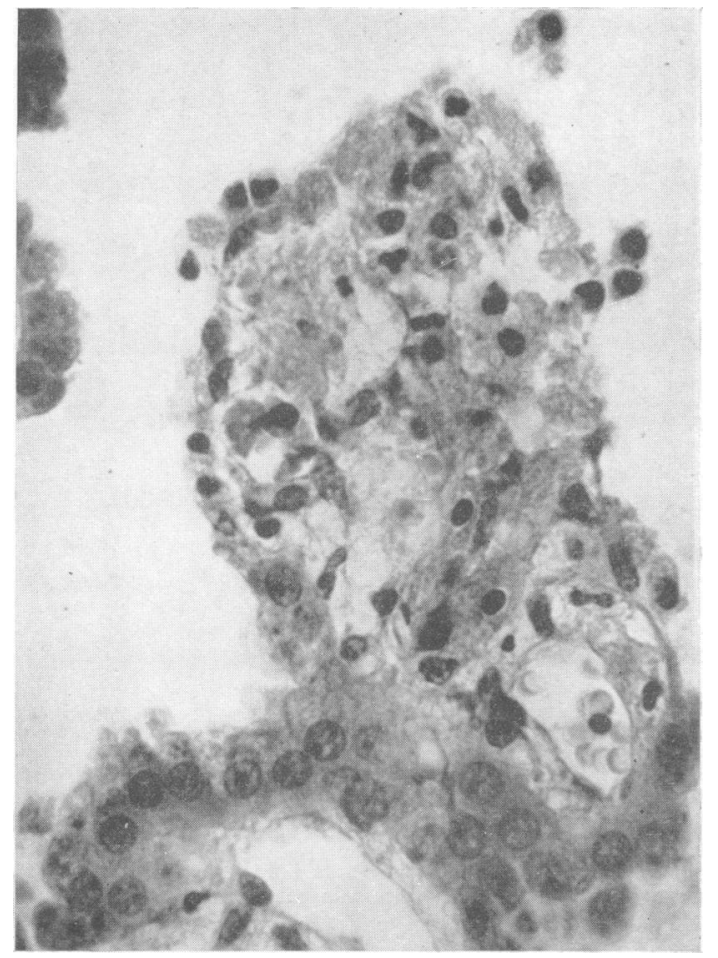

FIG. 3.-Necrosis of a tuft of the choroid plexus in the lateral ventricle. Single dose of neurotoxic goat serum. $\times 456$.

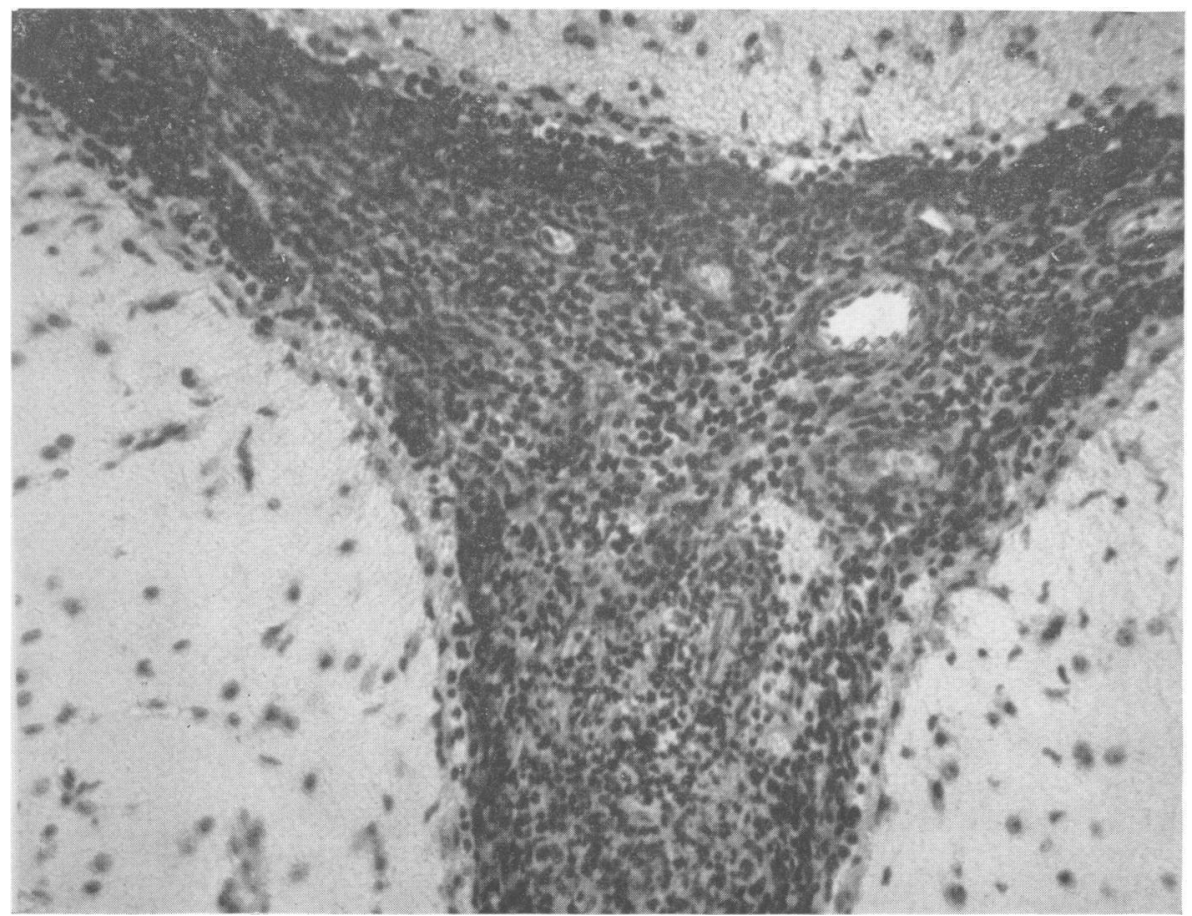

Frg. 4.-Meningitis in the Sylvian fissure. Multiple doses of neurotoxic goat serum. $\times 234$. 


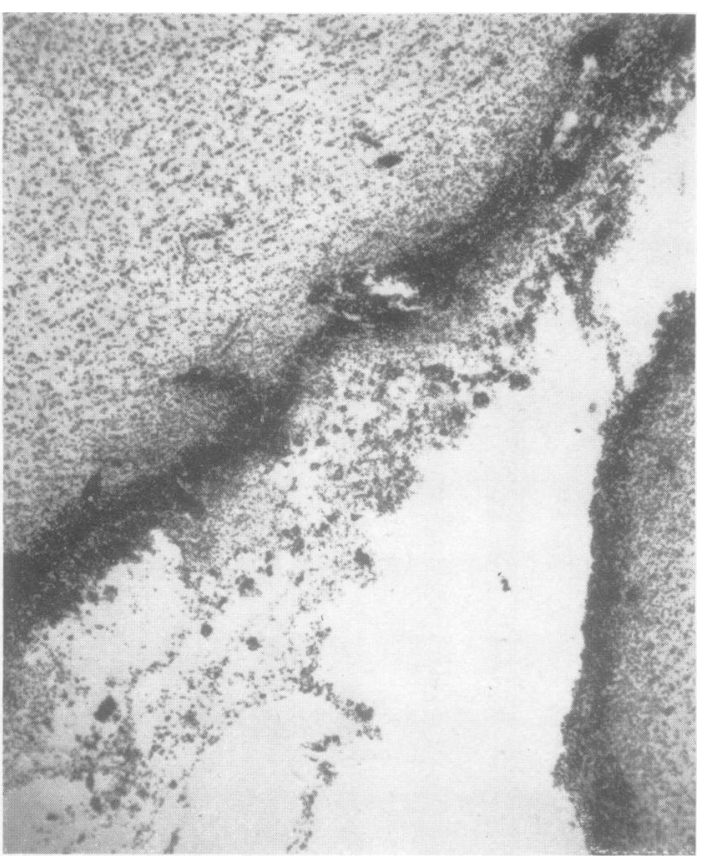

FIG. 5.-Zone of inflammatory tissue lining the lateral ventricls. Multiple doses of neurotoxic goat serum. $\times 140$.

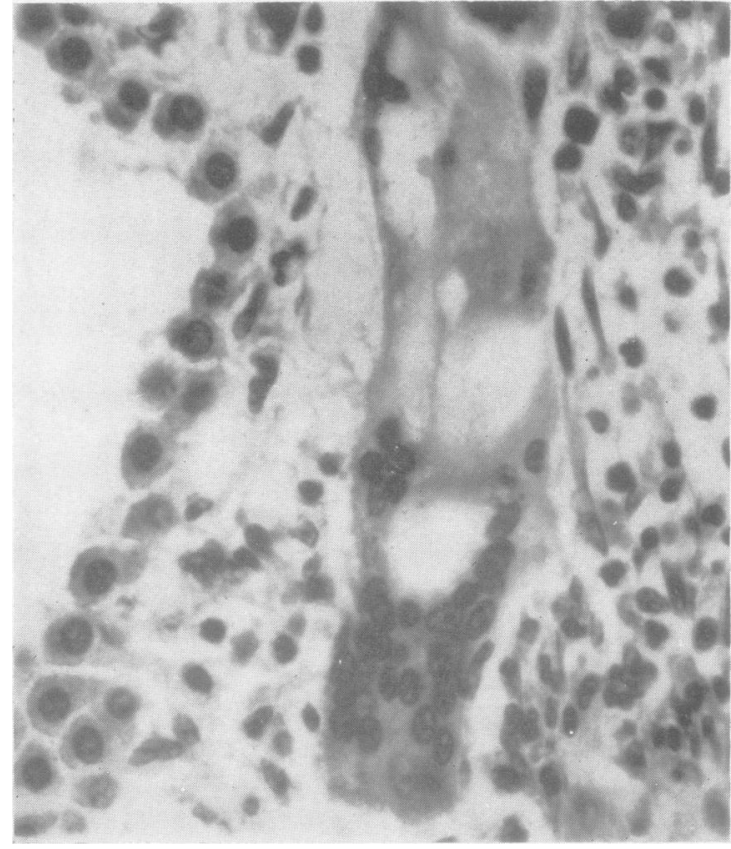

Fig. 7.-Large giant cell and inflammatory reaction in a choroid plexus. Multiple doses of neurotoxic goat serum. $\times 630$.

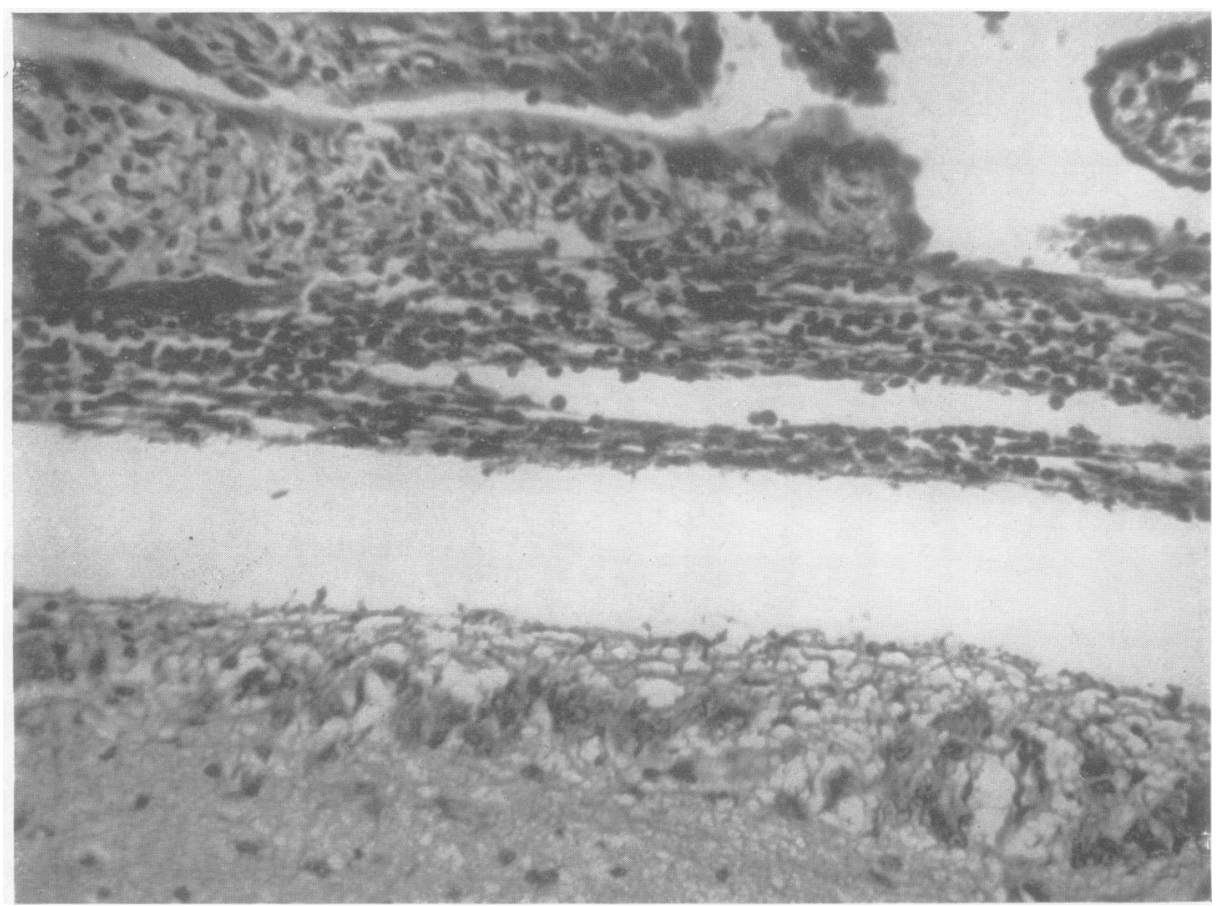

ГIG. 6.-Above, inflammation of the choroid plexus : below, palisade of enlarged glial cells beneath a surface denuded of ependyma in the lateral ventricle. Multiple doses of neurotoxic goat serum. $\times 325$. 
Morrison, 1951), in the recently immunized goats we removed the lymph nodes draining the areas receiving brain emulsion. A centrifuged $20 \%$ suspension of these lymph nodes, given at intervals of three to four days for two and four doses respectively intracisternally in two monkeys, failed to reproduce the condition seen with immune serum and caused only a mild and predominantly mononuclear meningitis.

One of six pigs receiving anti-porcine-brain rabbit serum presented ventricular changes similar to those described above, except that perivascular infiltration occurred at greater depth from the surface of ventricle or meninges. The remainder, together with pigs receiving anti-porcine-brain sheep serum, sheep receiving anti-sheep-brain rabbit serum, and monkeys receiving anti-simian-brain rabbit serum showed meningeal infiltration of varying intensity and nearly always more severe than that produced by the corresponding control serum ; they did not, however, suffer the ventricular lesions. Some of the sera were the outcome of prolonged immunization with the foreign brain material (without adjuvants), and we received the impression that not all animal species are equally suitable as producers of a neurotoxic serum. As far as simian brain is concerned, the goat appears to be a very suitable animal.

Monkeys receiving 1.5 to $3.0 \mathrm{ml}$. anti-spleen serum and dying within four hours exhibited intense congestion of the meningeal vessels, with many small haemorrhages in places. A massive polymorphonuclear exudate already filled the meshes of the pia-arachnoid over the brain-stem, the cerebellum, the greater part of the lateral surface of the cerebrum, and the upper part of the medial surface ; it continued for short distances, rarely exceeding the depth of the molecular zone of the cortex, along the vessels penetrating the nervous substance. In a few foci polymorphs were numerous also in oedematous areas of the molecular zone, especially along the heavily involved Sylvian fissure. The stroma of the choroid plexus of the fourth ventricle was similarly and densely infiltrated, but upper parts of the ventricular system escaped damage.

With single or repeated doses of anti-spleen serum diluted 1 in 2 or 1 in 4 with saline, lesions were generally similar but milder. The surface of the brains were always more congested than with neurotoxic serum. After three doses of 1 in 2 serum in six days followed by an unsuccessful puncture four days later, a monkey showed a mixed polymorphonuclear and mononuclear meningitis ; where lesions occurred in the superficial zone of the cerebral cortex, the cells were now mainly microglial.
In the ventricular system lesions were seen only in the fourth ventricle and were here confined to the stroma of the choroid plexus, leaving the choroidal epithelium, ependyma, and nervous tissues intact. The freedom from change in the upper parts of the ventricular system was no doubt associated with the relatively small inocula-each of $2 \mathrm{ml}$.- - but it appeared also that qualitatively the anti-spleen serum differed from the neurotoxic serum in its negligible effect on the nervous structures proper.

After injection of normal goat serum on one or more occasions, monkeys showed by comparison with the above a very mild and predominantly mononuclear infiltration of the meninges and choroid plexuses. They suffered no loss of the ependyma or of the choroidal epithelium.

\section{Comment}

It is clear that the serum of goats immunized with simian brain, whether by long-continued massive injections or more rapidly by modern techniques utilizing adjuvants, acquires the property of exciting very severe nervous lesions when injected intracisternally into monkeys. Neurotoxic sera produced in other species (none, it should be noted, by modern techniques) were less spectacular in their effects, a fact which may perhaps explain in part the failure of other modern investigators to obtain lesions with the sera of animals suffering from " allergic encephalitis". The lesions we describe differ in several important respects from those produced by a variety of chemical irritants introduced intracisternally (see Hurst, 1955); they presumably represent the effects of an antigenantibody reaction occurring at the sites affected, which are wholly superficial at points in the meninges or on the ventricular surface with which the serum is likely to come in contact. Some of the histological features in animals receiving multiple doses, and in particular the presence of numerous eosinophils and giant cells, are compatible with this assumption. An anti-spleen serum produced in the goat appeared quantitatively more toxic than the anti-brain sera but qualitatively different in attacking only mesodermal structures.

While thus the claims of older writers to have produced sera toxic to the nervous system have been upheld, the present observations make no contribution to the knowledge of how "allergic encephalitis" arises or of whether or how allergy is concerned in the aetiology of the demyelinating diseases of man and animals. Even if a common factor were involved in the production of "allergic encephalitis" on the one hand and of the lesions here described on the other, experiments such as we have 
conducted might well fail to bring it to light in view of the highly artificial conditions pertaining to the route of introduction of antibody, concentration at the time of exposure to antigen, and so on. There is, however, no compelling reason to associate the two. The development of " allergic encephalitis" has been linked with the development of sensitivity of the tuberculin type, while many writers witness to the inconstancy and variety of circulating antibodies which may follow immunization of animals with a highly complex system such as nervous tissue. On general grounds it seems more than likely that the lesions we describe are produced by a mechanism different from that responsible in "allergic encephalitis" but further work is needed to substantiate this view.

\section{Summary}

Immunization of goats with simian brain resulted in sera with neurotoxic properties. The lesions produced in monkeys receiving these sera intrathecally are described and compared with those following injection of an anti-spleen serum or of normal goat serum. Anti-brain sera produced in rabbits or sheep with the brains of several species were less toxic when introduced intrathecally into the donor species than were those derived from goats.

\section{REFERENCES}

Armand-Delille, P. F. (1906). Ann. Inst. Pasteur, 20, 838

Centanni, E. (1900). Rif. med., 16 (4), 374.

Délezenne, C. (1900). Ann. Inst. Pasteur, 14, 686.

Freund, J., and McDermott, K. (1942). Proc. Soc. exp. Biol., N. Y., $49,548$.

, Stern, E. R., and Pisani, T. M. (1947). J. Immunol., 57, 179. Hill, K. R. (1949). Bull. Johns Hopk. Hosp., 84, 302.

Hurst, E. W. (1944). Brain, 67, 103.

(1952). Amer. J. Med., 12, 547.

(1955). J. Path. Bact. In the press.

Innes, J. R. M. (1951). J. comp. Path., 61, 241.

Kabat, E. A., Wolf, A., and Bezer, A. E. (1948). J. exp. Med., 88,417 .

Morgan, I. M. (1947). Ibid., 85, 131

Pirone, R. (1907). Arch. Sci. biol., St. Pétersb., 10, 75.

Schmidt, A. (1906). Ann. Inst. Pasteur, 20, 601.

Waksman, B. H., and Morrison, L. R. (1951). J. Immunol., 66, 421. 